\title{
Damage Characterization Using the Extended Finite Element Method for Structural Health Management
}

\author{
T. Krishnamurthy ${ }^{*}$ \\ NASA Langley Research Center, Hampton, VA 23681, U.S.A. \\ and \\ Adam M. Gallegos \\ Lockheed Martin Information Systems and Global Solutions, Hampton, VA 23681, U.S.A.
}

\begin{abstract}
An efficient damage characterization method using the Extended Finite Element Method (X-FEM) combined with an optimization procedure is developed and demonstrated. The procedure developed can be used to characterize the damage size and location in a structural health management system. The method is computationally efficient, since it uses a single finite element model without requiring a different mesh for each damage configuration. The damage is characterized by the damage size, the damage location and the damage orientation angle. Numerical examples are presented to demonstrate the procedure in several damage configurations. The procedure estimates the damage size, the location and the orientation angle accurately in all the examples.
\end{abstract}

\section{Introduction}

The development of validated multidisciplinary Integrated Vehicle Health Management (IVHM) technologies to enable detection, diagnosis, prognosis, and mitigation of adverse conditions during flight will provide effective solutions to deal with safety-related challenges facing next generation aircraft. The adverse conditions include loss of control caused by environmental factors, actuator and sensor faults or failures, and damage conditions. A major concern in these structures is the growth of undetected damage (i.e., cracks) due to fatigue and low velocity foreign object impact that can reach a critical size during flight, resulting in loss of control of the aircraft. Hence, development of efficient methodologies to determine the presence, location, and severity of damage in critical structural components is highly important in developing efficient structural health management systems.

Approaches for detection of damage size and location in structures can be characterized as either global approaches or local approaches. In the global approach, changes in vibration properties caused by damage are used to detect its size and location [1, 2]. In the local approach, changes in the characteristics of ultrasonic waves propagating across damage are measured and analyzed to detect and characterize damage [3]. The global approaches are only effective for detecting larger damage, since smaller damage size may have only negligible effect on vibration properties. The local approach is effective in detecting smaller damage, but generally requires a dense network of sensors. Even with the continuous advancement in global and local approaches, there still are large uncertainties associated with determination of the damage size and the location. The uncertainties come from both the method employed and the instrumentation which results in low confidence in estimating the damage size and location. Hence there is a need to increase the level of confidence in detecting the damage size and location. One way to increase the confidence in detecting the damage size and location is to use another method to validate the results obtained from the global and local approach described earlier. In this paper, a finite element based method combined with the measured displacement field from the sensors is used to determine the damage size and location. Since there is no unique parameter (like stress intensity factor or crack opening angle) which can characterize the damage size and location, the displacement-based method alone is not sufficient to detect the damage. Hence, information from the global and local approaches is also needed to supplement the displacement-based damage detection methods.

\footnotetext{
* Aerospace Engineer, Durability and Damage Tolerance, Associate Fellow, AIAA
} 
Recent developments in Fiber Optics Strain Sensing (FOSS) technology offer the ability to obtain denselydistributed strain measurement with low weight addition to the structures [4]. The large area of strain measurement from FOSS technology can be used to obtain the displacement field around the damage in the structure [5]. These displacement fields measured from FOSS technology can be used in the damage characterization methods for Structural Health Management (SHM). The current research uses the displacement measured at selected points in the structure along with extended finite element analysis of the damaged structure to characterize the damage location and size. The eXtended Finite Element Method (X-FEM) is a standard displacement-based finite element approximation with enriched additional (special) functions at selected finite element nodes surrounding the damage [6]. The X-FEM is more computationally efficient than the conventional finite element analysis for damage propagation studies, since there is no need to model the actual damage in the analysis.

In this paper, a damage characterization method using the extended finite element method for Structural Health Management (SHM) is proposed. First, a brief introduction to the X-FEM is presented. Then, an optimization procedure is presented to estimate the damage size and location. Next, several numerical examples are presented to demonstrate the effectiveness of the method. Finally, a brief summary is presented.

\section{Extended Finite Element Method (X-FEM)}

The X-FEM provides significant benefits in the numerical modeling of damage propagation compared to the conventional finite element method. In the conventional finite element method, the existence of damage is modeled by requiring the damage to follow element edges. In contrast, the damage geometry in the X-FEM need not be aligned with the element edges, and there is no need to model the physical damage. The ability to place an arbitrary damage without explicitly modeling the damage in the X-FEM provides flexibility and versatility in the modeling. The method is based on the enrichment of the Finite Element (FE) model with additional degrees of freedom (DOFs) that are tied to the set of nodes on which the damage has its influence (support) domain. The displacement for the nodes belonging to the damage influence (support) domain can be expressed as

$$
V=V^{F E}+V^{E n r}
$$

where

$$
\begin{array}{ll}
V & \text { - displacement for the support domain nodes } \\
V^{F E} & \text { - displacement from conventional finite element } \\
V^{E n r} & \text { - displacement from enrichment added to the nodes due to the damage }
\end{array}
$$

The enriched function $V^{E n r}$ in Equation 1 consists of functions to represent the displacement discontinuity across the damage faces and also the singularity due at the damage tips. For example, Figure 1 shows a portion of the finite element mesh with 4-node quadrilateral elements. The location of the arbitrary damage is shown as a thick solid line in Figure 1. Note that the damage is not modeled physically in the model. The nodes marked with filled circles are nodes enriched with special functions due to the presence of the damage. The information about the enriched nodes is the only additional information provided for the damage analysis in the X-FEM. The description of the type of enriched functions used in the X-FEM is beyond of the scope this paper. Interested persons can read more about the enriched special functions and their implementation for the X-FEM from the book on the Extended Finite Element Method [6]. The X-FEM is currently available in the general purpose finite element structural analysis code ABAQUS $^{\circledR}[7]^{\dagger}$ and is used for structural analysis in this paper.

\footnotetext{
${ }^{\dagger}$ Trade names and trademarks are used in this report for identification only. Their usage does not constitute an official endorsement, either expressed or implied, by the National Aeronautics and Space Administration.
} 


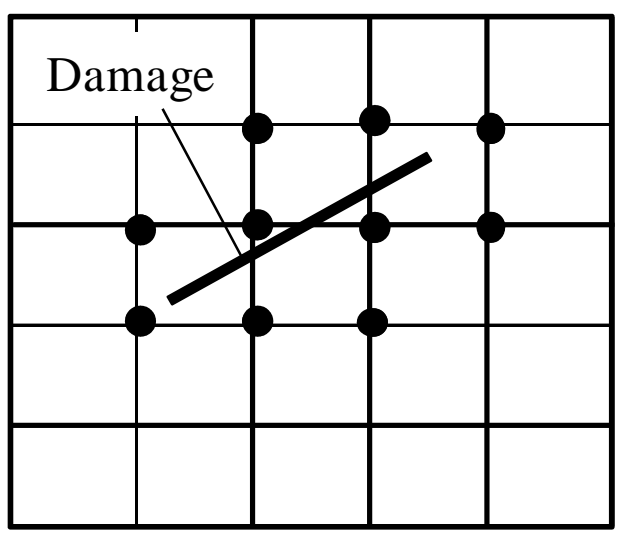

- Enriched Nodes

Figure 1. Enriched nodes in the X-FEM: Nodes with filled circle represent the enriched nodes.

\section{Parameters to characterize the damage size and location}

For the present study, damage in a two-dimensional geometry is considered. The damage size and location are characterized by three parameters in a two-dimensional plate with height $h$ and width $w$ as shown in Figure 2. The parameters are the location of the center of the damage $\left(X_{c}, Y_{c}\right)$, the damage size $(a)$, and the damage orientation angle $(\theta)$ made by the damage with respect to the $x$-axis. In the present paper, in order to minimize the number of parameters to describe the damage, the damage location is characterized by the distance $Y_{c}$ only, thus ignoring the distance $X_{c}$. The next section will describe the optimization procedure used to characterize the damage.

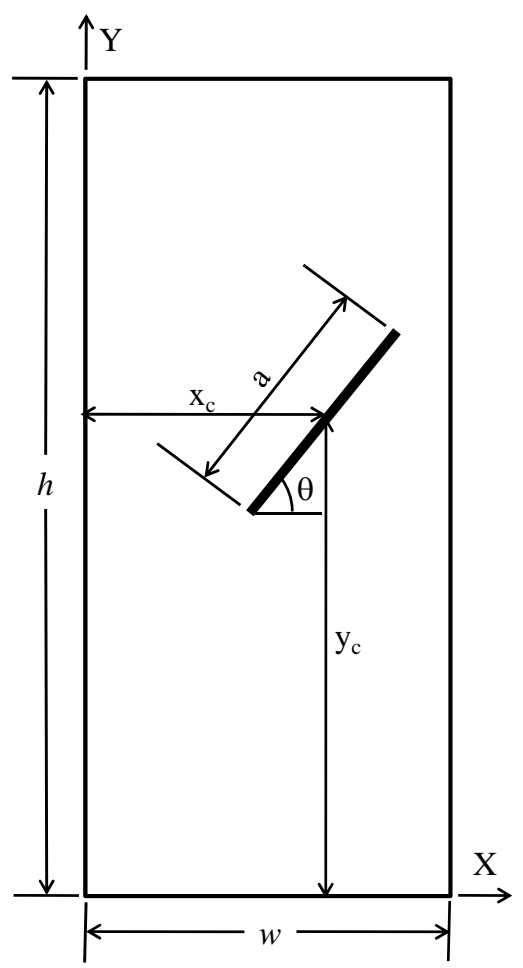

Figure 2. Parameters to characterize the damage 


\section{Optimization Procedure to Characterize the Damage}

An optimization procedure to characterize the damage size and the location is described in this section. The accuracy and reliability of the damage size and location largely depend on the number of points where the displacement values are available for the structure. In general, larger the number of points the displacement values is known, the greater the accuracy is of the detected size and location. For the present study, it is assumed that the displacement values are known at all the points in the finite element model, and these displacement values are the reference solution. Using the reference solution, the process of estimation of damage size and location is initiated with a finite element model of the structure. Then, an X-FEM fracture analysis is performed on the finite element model for an assumed damage size and location. If the assumed damage size and location are correct, the displacement field from the X-FEM analysis will accurately match the reference solution. If not, the X-FEM analysis is repeated for a different damage size and location. The iterations are continued until the displacement field for the current assumed damage size and location matches the reference solution. The number of iterations needed for the convergence of the solution is minimized by the optimization procedure described below:

1. Construct a finite element model for the flight vehicle structure.

2. Generate the reference displacements at all the nodal locations of the finite element model. Note that in the actual flight vehicle structure, the reference displacements are obtained at as many points as possible from the sensor locations. However, for the present study the reference displacements are obtained from a finite element analysis with the damage.

3. Assume an arbitrary damage location and size.

4. Perform an X-FEM analysis and obtain the nodal displacements.

5. Find the sum of the squares of the error between the X-FEM and reference displacements as

$$
\phi=\sum_{i=1}^{N}\left(V_{R e f}-V_{X-F E M}\right)^{2}
$$

Where

$$
\begin{array}{ll}
V_{\operatorname{Re} f} & \text { - displacements for the flight vehicle structure } \\
V_{X-F E M} & \text { - displacements from the X-FEM analysis for the current damage location and size } \\
N & \text { - number of nodes in the X-FEM model }
\end{array}
$$

6. Estimate the function $\phi$ in Equation 2 by finding the difference in displacements between the flight vehicle structure and the current X-FEM.

7. If the convergence in minimizing $\phi$ is achieved in the optimization procedure, the iteration is stopped. If not, the optimization algorithm will estimate a new damage location and size and the Steps 4-7 are repeated until the convergence is achieved.

The optimization code DOT [8] is used in Steps 5 to 7 to minimize the difference in displacements in Equation 2. This procedure is also explained in the flow chart in Figure 3.

The above procedure is now shown to estimate the damage size, the location, and the orientation angle in the subsequent sections through numerical examples. First, the location is estimated keeping the damage size and the angle constant. Next, the damage location and the size are estimated keeping the orientation angle constant. Lastly, all the three parameters, the location, the size and the orientation angle, are estimated. 


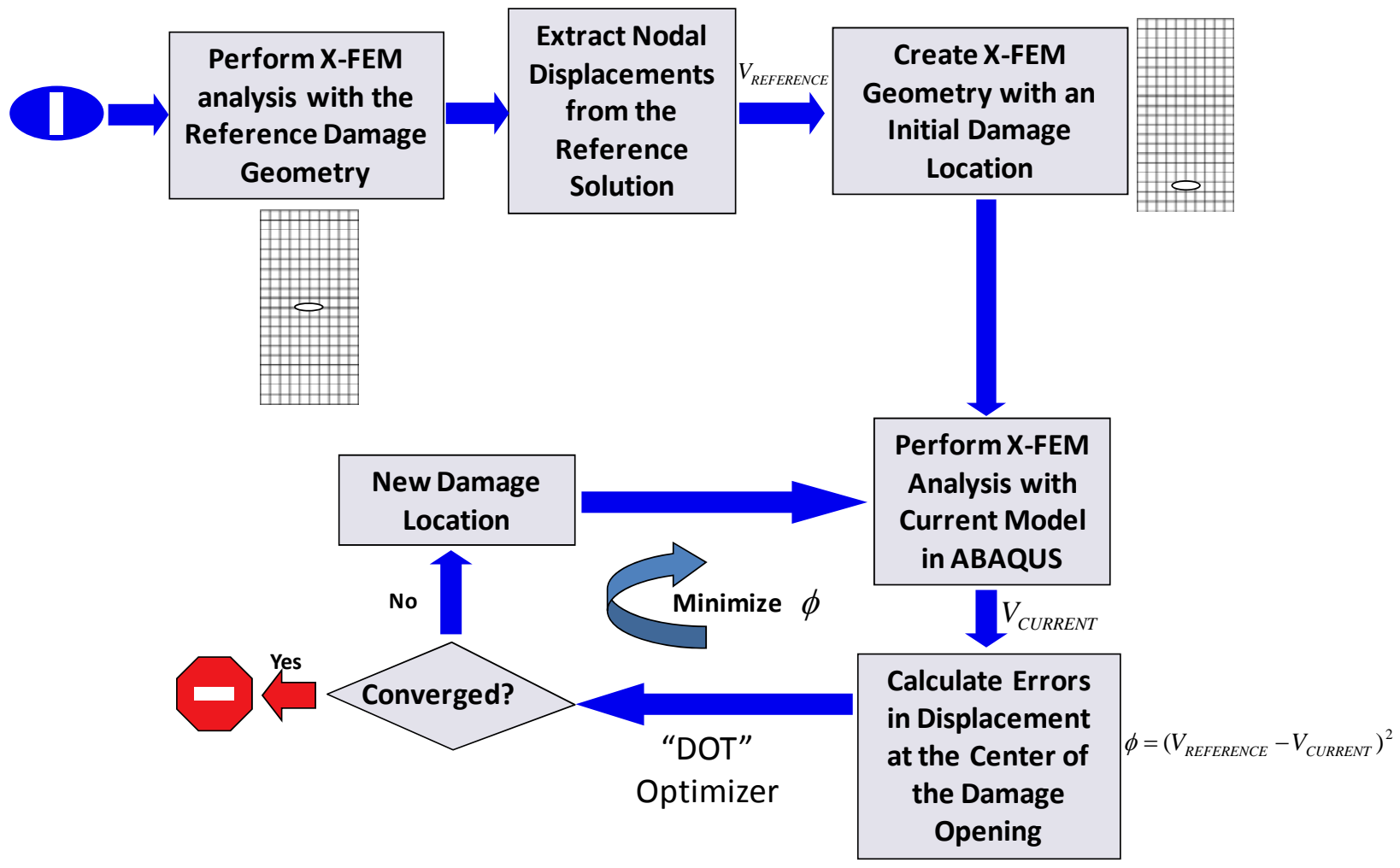

Figure 3. Optimization procedure for determining the damage size and location.

\section{Numerical Examples}

The procedure described in Section III for damage characterization using the X-FEM is demonstrated with a center damage located in a two-dimensional plate. The plate geometry and material properties along with the finite element model used in the analyses are shown in Figure 4.

$E=10 E 10^{6} P S$

$v=0.3$

Elements $=115200$ (4-Node Quad)

Nodes $=115921$

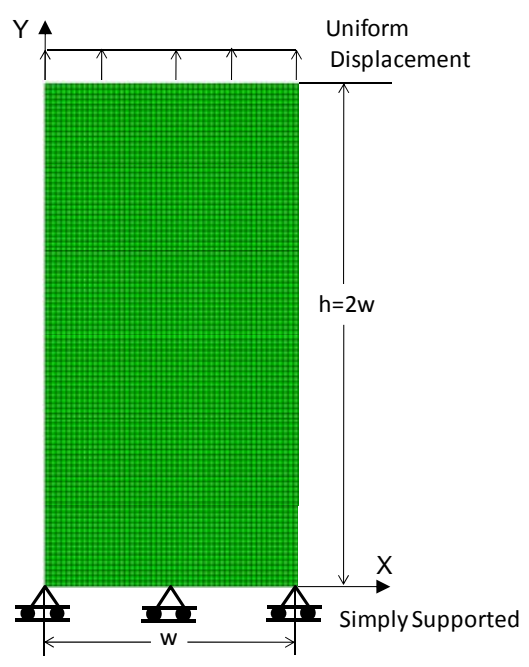

Figure 4. Finite element model for X-FEM analysis 


\section{a. Estimation of location of damage for a fixed damage size and orientation angle:}

For estimating the location of the damage, the size $(a)$ and the orientation angle $(\theta)$ are fixed. For the numerical examples presented, the damage size is fixed at $a=0.167 \mathrm{w}$ and the orientation angle is fixed at $\theta=0$ degrees. Two different damage locations are considered for the reference solution as shown in Figure 5.
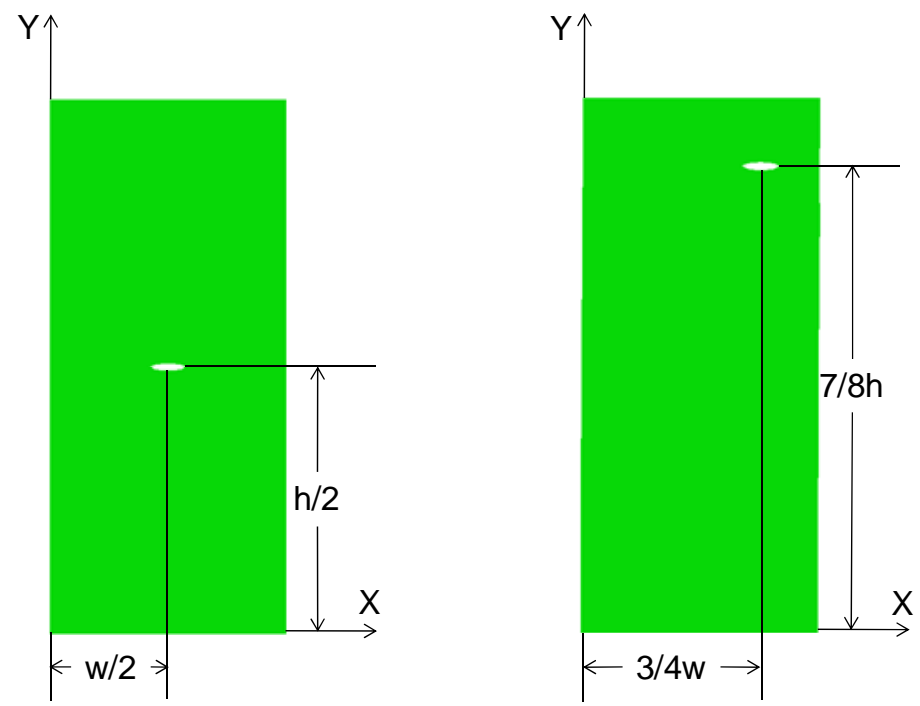

Figure 5. Damage locations analyzed for the reference solution

Initial analyses with the optimization procedure concluded that the accuracy of the location estimation is sensitive to the mesh size (element size) used for the X-FEM. Hence a mesh size sensitivity study was performed. The results from the study are shown in Table 1. The mesh size was varied from 2.5 percent to 10 percent of the damage size. As seen from Table 1, a mesh size less than five percent of the damage size gives estimates of the damage location to within 1 percent. However initial experiments with X-FEM for the other cases presented in the paper, suggested that the mesh size of 2.5 is most well suited for all the examples. Hence, the mesh size equal to 2.5 percent of the damage size was used in all the examples presented in this paper.

Table 1: Mesh size sensitivity for estimation of damage location

\begin{tabular}{|c|c|c|}
\hline $\begin{array}{c}\text { Mesh Size } \\
\text { (\% of Damage Length) }\end{array}$ & $\begin{array}{c}\text { Predicted Y Location of } \\
\text { Damage }\end{array}$ & $\begin{array}{c}\text { Difference from } \\
\text { Reference Solution }\end{array}$ \\
\hline 10 & $0.118 \mathrm{~h}$ & $5.59 \%$ \\
5 & $0.1248 \mathrm{~h}$ & $0.15 \%$ \\
2.5 & $0.1248 \mathrm{~h}$ & $0.13 \%$ \\
\hline
\end{tabular}

First, the damage location for the reference solution is assumed at the center of the plate. The optimization procedure described in Section III is initiated each time with a different initial damage location. The final location of the damage is determined from the X-FEM analyses and the optimization procedure. The converged locations of the damage for different initial damage locations are shown in Figure 6 for damage at the center of the plate as the reference solution. It can be observed that the final damage location is within 1.5 percent of the reference damage location irrespective of the initial starting damage location. 


\begin{tabular}{|c|c|c|}
\hline $\begin{array}{c}\text { Initial Guess } \\
\text { YLocation of Damage }\end{array}$ & $\begin{array}{c}\text { Predicted Y Location } \\
\text { of Damage }\end{array}$ & $\begin{array}{c}\text { Difference from } \\
\text { Reference Solution }\end{array}$ \\
\hline $0.125 \mathrm{~h}$ & $0.4975 \mathrm{~h}$ & $0.50 \%$ \\
$0.25 \mathrm{~h}$ & $0.4997 \mathrm{~h}$ & $0.07 \%$ \\
$.75 \mathrm{~h}$ & $0.4932 \mathrm{~h}$ & $1.36 \%$ \\
$.875 \mathrm{~h}$ & $0.4959 \mathrm{~h}$ & $0.82 \%$ \\
\hline
\end{tabular}

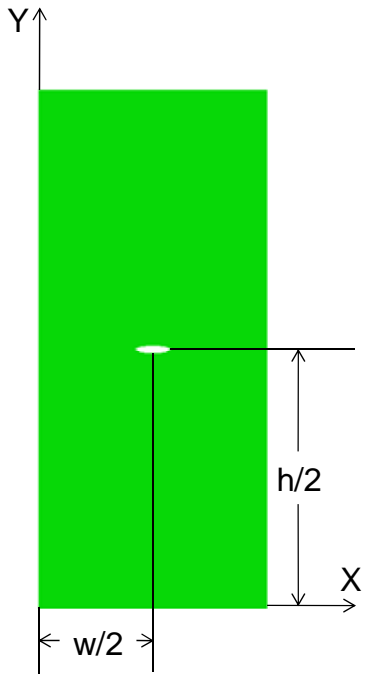

Figure 6. Estimation of damage location: optimization solution convergence study

The displacement distribution for a typical case (initial guess damage location at $0.125 h$ ) from Figure 6 is shown along with the reference solution in Figure 7. It is seen from the figure that the final optimized solution deformation is similar to the reference solution deformation.

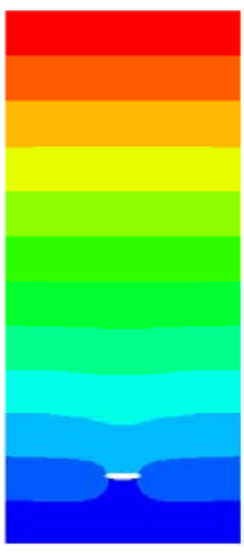

Initial Guess Damage Location

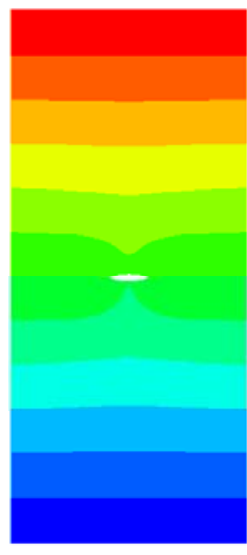

After Optimization

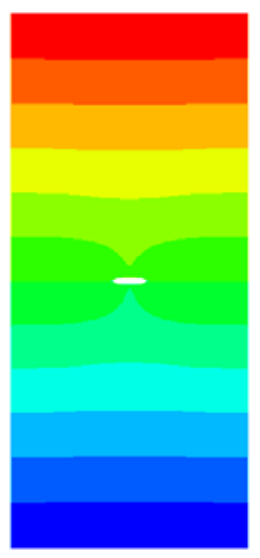

Reference Solution

Figure 7. Displacement distribution: final predicted solution with reference solution

Similarly, the converged locations of the damage for different initial damage locations are shown in Figure 8 for an offset damage near the top edge of the plate as the reference solution. It can be observed that the final damage location is within 3.0 percent of the reference damage location irrespective of initial location. 


\begin{tabular}{|c|c|c|}
\hline $\begin{array}{c}\text { Initial Guess } \\
\text { Y Location of Damage }\end{array}$ & $\begin{array}{c}\text { Predicted Y Location } \\
\text { of Damage }\end{array}$ & $\begin{array}{c}\text { Difference from } \\
\text { Reference Solution }\end{array}$ \\
\hline $0.125 \mathrm{~h}$ & $0.900 \mathrm{~h}$ & $2.86 \%$ \\
$0.25 \mathrm{~h}$ & $0.8763 \mathrm{~h}$ & $0.12 \%$ \\
$0.5 \mathrm{~h}$ & $0.8748 \mathrm{~h}$ & $0.03 \%$ \\
$0.75 \mathrm{~h}$ & $0.8717 \mathrm{~h}$ & $0.39 \%$ \\
\hline
\end{tabular}

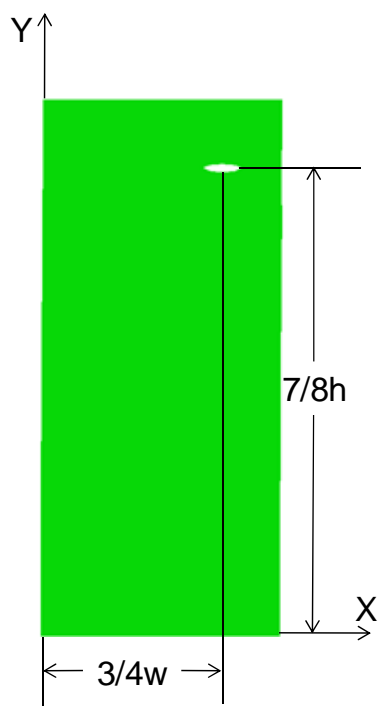

Figure 8. Damage near the top edge of the plate: optimization solution convergence study

The displacement distribution for a typical case (initial guess damage location at $0.125 \mathrm{~h}$ ) from Figure 8 is shown along with the reference solution in Figure 9. It is seen from the figure that the final optimized solution deformation is similar to the reference solution deformation.

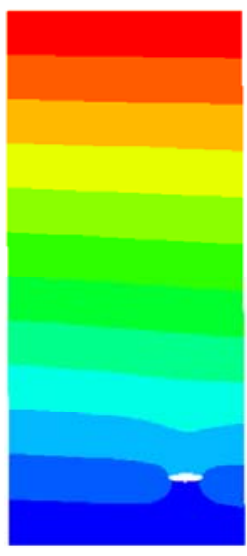

Initial Guess Damage Location

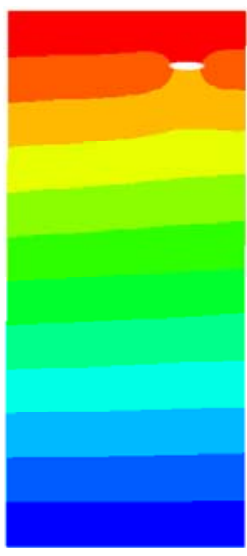

After Optimization

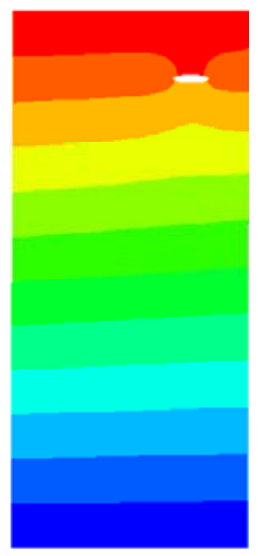

Reference Solution

Figure 9. Displacement distribution: final predicted solution with reference solution

The two numerical examples demonstrated that the optimization procedure developed here can predict the damage location accurately for all damage configurations. Next, both the damage size and location are estimated keeping the orientation angle constant.

\section{b. Estimation of damage size and location for a fixed orientation angle:}

In order to demonstrate the optimization procedure for estimating the damage size and location for a given orientation angle, the center damage in the plate, as shown in Figure 5, was selected. The optimization procedure described in Section III is initiated each time with a different damage size and location. The final location of the damage is determined from the X-FEM analyses and the optimization procedure. Initial guesses for the damage location were selected as $0.25 \mathrm{~h}$ and $0.75 \mathrm{~h}$. For each of the initial damage locations, four different damage sizes, $0.083,0.167,0.333$ and 0.667 were considered. The results from the optimization procedure convergence study are presented in Figure 10. 


\begin{tabular}{|c|c|c|c|}
\hline $\begin{array}{c}\text { Initial Guess } \\
\text { Y Location of Damage }\end{array}$ & $\begin{array}{c}\text { Optimized Y Location } \\
\text { Size of Damage } \\
(\mathrm{a} / \mathrm{W})\end{array}$ & $\begin{array}{c}\text { Optimized Size of } \\
\text { of Damage } \\
\text { Difference from } \\
\text { Reference Solution }\end{array}$ & $\begin{array}{c}\text { Dafference from } \\
\text { Reference Solution }\end{array}$ \\
\hline $0.25 \mathrm{~h}$ & 0.083 & $0.01 \%$ & $7.75 \%$ \\
$0.25 \mathrm{~h}$ & 0.167 & $0.03 \%$ & $6.98 \%$ \\
$0.25 \mathrm{~h}$ & 0.333 & $0.06 \%$ & $0.84 \%$ \\
$0.25 \mathrm{~h}$ & 0.667 & $0.03 \%$ & $3.42 \%$ \\
\hline $0.75 \mathrm{~h}$ & 0.083 & $0.09 \%$ & $4.32 \%$ \\
$0.75 \mathrm{~h}$ & 0.167 & $0.33 \%$ & $1.29 \%$ \\
$0.75 \mathrm{~h}$ & 0.333 & $0.06 \%$ & $3.31 \%$ \\
$0.75 \mathrm{~h}$ & 0.667 & $0.11 \%$ & $4.33 \%$ \\
\hline
\end{tabular}

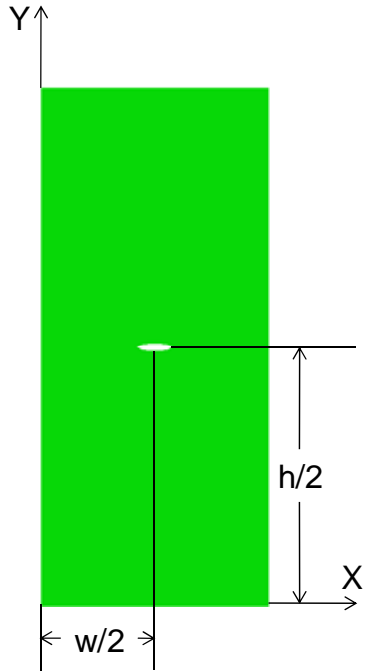

Figure 10. Estimation of damage size and location: optimization solution convergence study

It can be seen from Figure 10 that the damage locations are estimated within one percent, whereas the damage sizes are estimated within eight percent.

The displacement distribution for a typical case (initial guess the damage location at $0.25 \mathrm{~h}$ and the damage size $a=0.083 \mathrm{w}$ ) from Figure 10 is shown along with the reference solution in Figure 11. It is seen from the figure that the final optimized solution deformation is similar to the reference solution deformation.

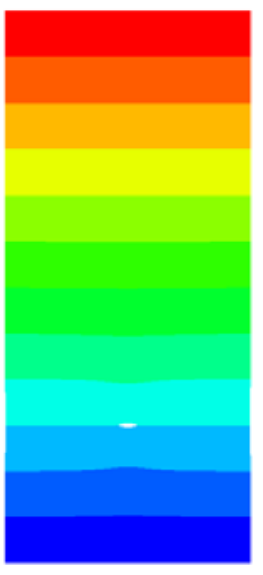

Initial Guess Damage Location

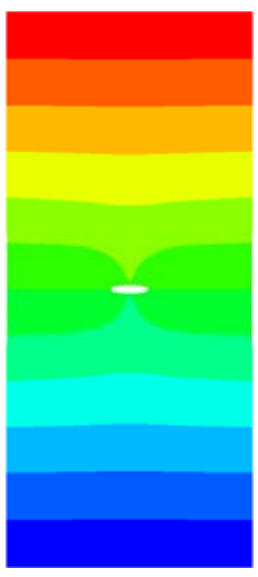

After Optimization

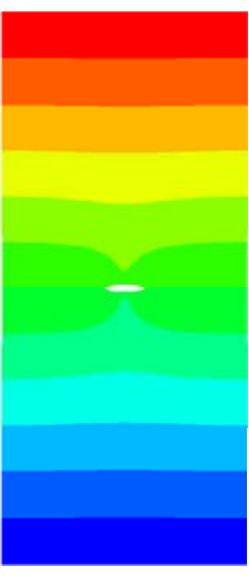

Reference Solution

Figure 11. Displacement distribution: final predicted solution with reference solution

The numerical examples presented clearly demonstrate that the optimization procedure combined with X-FEM is able to estimate the damage size and location accurately from the deformation information. Next, all three parameters (the damage size, the location, and the orientation angle) are estimated. 


\section{c. Estimation of the damage size, location and orientation angle:}

During the initial experimentation, it was difficult to obtain a converged solution from the optimization procedure when all three parameters (the damage size, the location, and orientation angle) were treated as unknowns. The reason for not obtaining a converged solution is under investigation. Hence, in the examples presented in this section, the orientation angle was fixed at either 45 or 65 degrees to enable estimation of damage size and the location. The results are presented next.

The results obtained for the orientation angle $\theta=45$ degrees (for reference damage size $a=0.167 \mathrm{w}$ and location $Y_{c}=0.5 h$ ) are shown in Figure 12. The optimization procedure was initiated with damage at location $Y_{c}=0.25 h$ and damage size of $a=0.153 \mathrm{~W}$. It can be seen from Figure 11 that the optimization procedure predicts the damage location within one percent and the damage size within two percent.

\begin{tabular}{|c|c|c|c|}
\hline $\begin{array}{c}\text { Initial Guess } \\
\text { Y Location of Damage }\end{array}$ & $\begin{array}{c}\text { Initial Guess } \\
\text { Size of Damage } \\
(\mathrm{a} / \mathrm{W})\end{array}$ & $\begin{array}{c}\text { Optimized Y Location } \\
\text { of Damage } \\
\text { Difference from } \\
\text { Reference Solution }\end{array}$ & $\begin{array}{c}\text { Optimized Size of } \\
\text { Damage } \\
\text { Difference from } \\
\text { Reference Solution }\end{array}$ \\
\hline $0.25 \mathrm{~h}$ & 0.153 & $0.06 \%$ & $1.67 \%$ \\
\hline
\end{tabular}

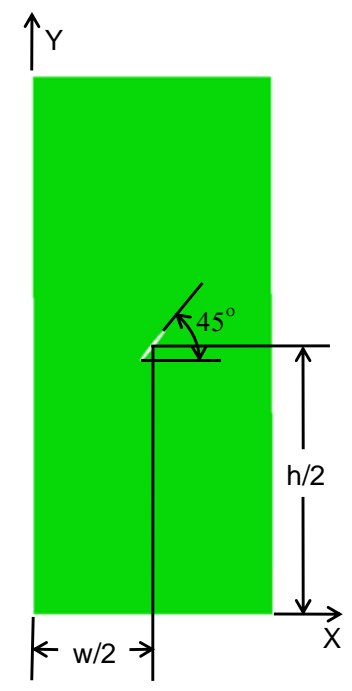

Figure 12. Estimation of damage size and location

The displacement distribution for the case from Figure 12 is shown along with the reference solution in Figure 13. It is seen from the figure that the final optimized solution deformation is similar to the reference solution deformation.

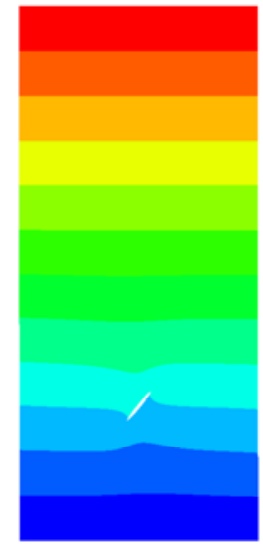

Initial Guess Damage Location

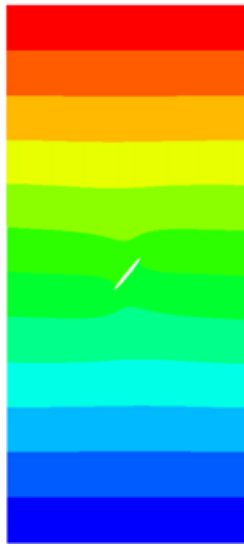

Predicted Solution

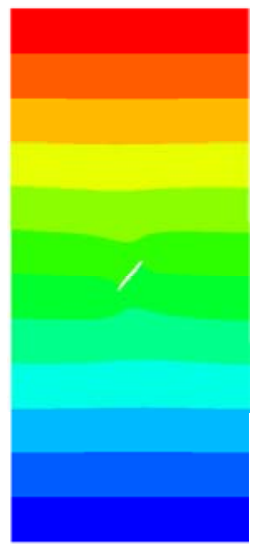

Reference Solution

Figure 13. Displacement distribution: final predicted solution with reference solution 
The results obtained for the orientation angle $\theta=65$ degrees (for reference damage size $a=0.167 \mathrm{w}$ and location $Y_{c}=0.5 \mathrm{~h}$ ) are shown in Figure 14. The optimization procedure was initiated with damage at location $Y_{c}=0.25 \mathrm{~h}$ and damage size of $a=0.153 w$. It can be seen from Figure 14 that the optimization procedure predicts the damage location within one percent and the damage size within two percent.

\begin{tabular}{|c|c|c|c|}
\hline $\begin{array}{c}\text { Initial Guess } \\
\text { Y Location of Damage }\end{array}$ & $\begin{array}{c}\text { Initial Guess } \\
\text { Size of Damage } \\
(\mathrm{a} / \mathrm{W})\end{array}$ & $\begin{array}{c}\text { Optimized Y Location } \\
\text { of Damage } \\
\text { Difference from } \\
\text { Reference Solution }\end{array}$ & $\begin{array}{c}\text { Optimized Size of } \\
\text { Damage } \\
\text { Difference from } \\
\text { Reference Solution }\end{array}$ \\
\hline $0.25 \mathrm{~h}$ & 0.153 & $0.58 \%$ & $1.22 \%$ \\
\hline
\end{tabular}

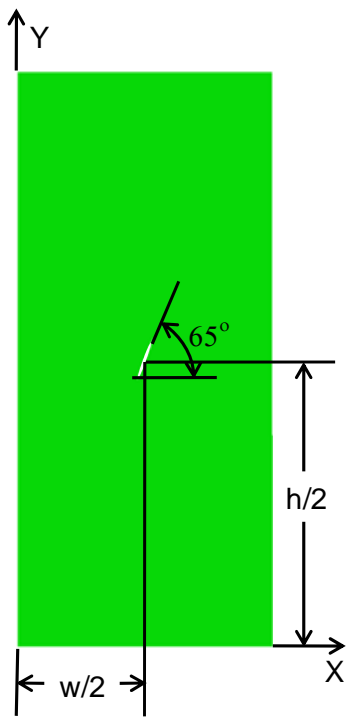

Figure 14. Estimation of damage size and location

The displacement distribution for case from Figure 14 is shown along with the reference solution in Figure 15. It is seen from the figure that the final optimized solution deformation agrees well with the reference solution.

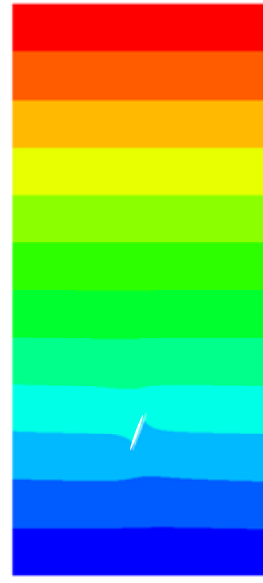

Initial Guess Damage Location

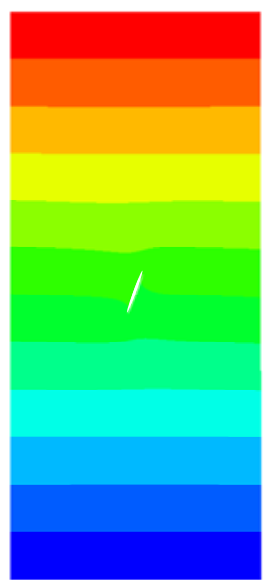

Predicted Solution

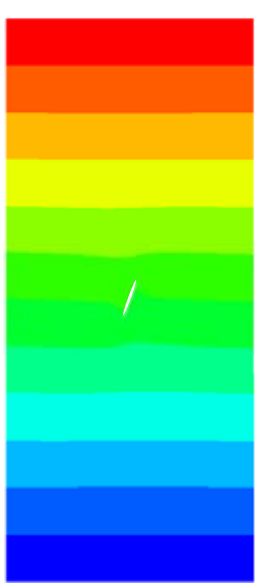

Reference Solution

\section{Figure 15. Displacement distribution: final predicted solution with reference solution}

The two numerical examples presented with nonzero orientation angles demonstrated that the damage size and the location can be estimated accurately for the orientation angles for which the optimization procedure is able to reproduce correct solutions. 


\section{Summary}

In this paper, an efficient damage characterization method using the Extended Finite Element Method (X-FEM) combined with an optimization procedure was developed and demonstrated. The procedure described can be used to characterize the damage size and location in a Structural Health Management system once fully developed. The XFEM method is computationally efficient, since it uses a single finite element model without requiring a different mesh for each damage configuration.

The damage is characterized by damage size, damage location and damage orientation angle. Numerical examples were presented to demonstrate the procedure in several damage configurations. First, the damage location was estimated keeping the damage size and orientation angle constant. It was found that the damage location is estimated within three percent for all the damage configurations tested. Next, both the damage size and the location were estimated keeping the orientation angle constant. It was found that the size and location are estimated within eight percent and three percent respectively for all the configurations analyzed. However in estimating all the three parameters (damage size, location and orientation angle), only limited success was achieved due to failure to obtain a converged solution from the optimization procedure. However, it was demonstrated that the damage size and the location can be estimated accurately for the orientation angles for which the optimization procedure is able to reproduce correct solutions.

\section{References}

1. Kim, J.T. and Stubbs, N., "Improved Damage Identification Method Based on Modal Information," Journal of Sound and Vibration, Vol. 252, pp. 223-238, 2002.

2. Mal, A.K., Ricci, F., Banerjee, S., and Shih, F., "A Conceptual Structural Health Monitoring System Based on Vibration and Wave Propagation," Structural Health Monitoring: An International Journal, Vol. 4, pp. 283 - 293, 2005.

3. Wang, L., and Yuan, F. G.," Active Damage Localization Technique Based on Energy Propagation of Lamb Waves," Smart Structures and Systems, Vol. 3, pp. 201-217, 2007.

4. Quach, C., Vazquez, S., Tessler, A., Moore, J., Cooper, E., and Spangler, J.," Structural Anomaly Detection using Fiber Optic Sensors and Inverse Finite Element Method," AIAA Guidance, Navigation, and Control Conference and Exhibit, San Francisco, CA. AIAA Paper 2005-6357, 2005.

5. Tessler, A., and Spangler, J.," A Least-Squares Variational Method for Full-Field Reconstruction of Elastic Deformations in Shear Deformable Plates and Shells," Computer Methods in Applied Mechanics and Engineering, Vol. 194, pp. 327-335, 2005.

6. Mohammadi, S., "Extended Finite Element Method for Fracture Analysis of Structures," by Blackwell Publishing Ltd., 2008.

7. Anonymous, Getting Started with ABAQUS ${ }^{\circledR}$, Version 6.9, ABAQUS, Inc., Providence, RI 02909.

8. Anonymous, DOT, Design Optimization Tools, User's Manual, Version 5.0, Vanderplaats Research \& Development, Inc., Colorado Springs, CO, 80906. 